\title{
Green light for neutrino beam to pass below the Alps
}

Geneva

A major project to send a beam of muon neutrinos from Geneva under the Alps to the Gran Sasso laboratories near Rome was approved last week by the council of CERN, the Geneva-based European Laboratory for Particle Physics.

Italy's INFN, its national organization for particle physics, is organizing two experiments, known as OPERA and ICANOE, to study the oscillations of the neutrinos during their 730-km trip to Gran Sasso.

The experiments are designed to extend the breakthrough results achieved at the SuperKamiokande (Super-K) experiment in Japan last year, which showed that a proportion of muon neutrinos from cosmic rays disappeared, presumed to have been converted to tau neutrinos, as the beam travelled through the Earth (see Nature 394, 13; 1998). The Gran Sasso experiments will be optimized to detect tau neutrinos directly.

The neutrino beamline will cost SFr71 million (US\$45 million), of which INFN will pay around two-thirds. Special donations from other CERN member states, including Germany, Switzerland and France, will provide SFr16 million. First experimental results will be collected in 2005 .

The idea has been under discussion for many years; Italian particle physicists have long been disappointed at being left behind. Japan and the United States are planning neutrino experiments using long-range

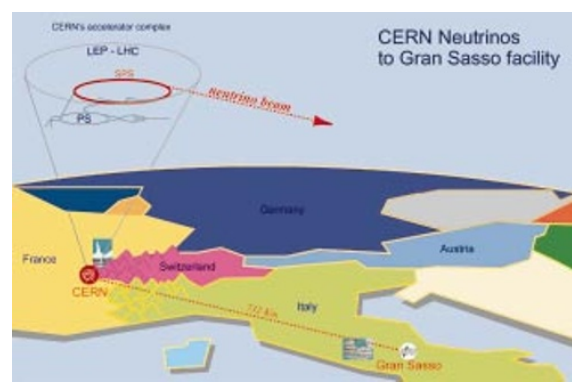

Direct route: the beam could solve some puzzles about neutrinos on its 2.5-millisecond journey.

accelerated beams in an attempt to repeat the Super-K results under more controlled conditions. But these will only look for disappearance of muon neutrinos, says Lorenzo Foa, professor of particle physics at the Scuola Normale Superiore, in Pisa, and a former director of research at CERN. "We will still need the positive confirmation of measuring the creation of tau neutrinos," he says, adding that Japan itself has expressed interest in participating in the experiments.

The approval also gives a shot in the arm to the INFN's laboratories in Gran Sasso. Built in the 1970s, they were deliberately orientated towards CERN in the hope that a neutrino beam would one day be created.

Luciano Maiani, the director-general of CERN, says that the quality of the planned neutrino beam and the innovative experiments being designed offer unique opportunities for science.

Alison Abbott
Scientists rally to defend schools against creationists

\section{San Francisco}

The American Geophysical Union (AGU) last week denounced the teaching of creationism and called for scientists to become politically involved in promoting the teaching of evolution.

The AGU council issued its 'advocacy statement' after a morning-long session at which scientists issued a call to arms to defend science teaching. More than 300 Earth scientists, including teachers, crowded into a session at the union's autumn meeting last week (see below), where they were urged to seek election to local school boards.

"The American Geophysical Union affirms the central importance of scientific theories of earth history and organic evolution in science education," reads the statement. "Creationism is not science and does not have a legitimate place in any science curriculum."

AGU president John Knauss said that creationists had become quite "clever" in distorting both science and the positions of scientific organizations. The union's previous statement was vulnerable in this regard, he added, because it was brief and did not sufficiently describe the AGU's opposition to creationism.

The AGU's new stance was prompted by the actions of two state school boards earlier this year. In Kansas, the board voted to cut the teaching of Earth science

\section{Geophysics union entices biologists with new section}

\section{San Francisco}

The American Geophysical Union (AGU) is setting up a new section for the biological sciences. The intention is to create a clearer focus and structure for incorporating biology into geophysical research and to attract biologists to the AGU.

Called 'Biogeochemistry, Biogeophysics and Planetary Ecosystems', the section is expected to advance research — particularly in global biogeochemical cycles and astrobiology - through scientific presentation posters, lecture meetings and publications.

It was approved by the AGU council during the union's annual autumn meeting last week in San Francisco. The meeting, attended by more than 7,500 scientists, included a biogeoscience programme that attracted more than 300 paper submissions. The council's required second vote is planned for the spring meeting in Washington DC.
A 13-member committee chaired by Diane McKnight of the University of Colorado had recommended creating the section. The panel said that improved coordination and articulation of the biological sciences "will foster a critical mass of researchers who will contribute the integrative links to advance our understanding of the Earth and planets".

The new section has been enthusiastically endorsed by AGU president John Knauss, a retired marine scientist associated with the University of Rhode Island and the Scripps Institution of Oceanography in San Diego, and president elect Marcia McNutt, a marine geophysicist who directs the Monterey Bay Aquarium Research Institute in California. "We must ensure that we are able to evolve as a society," said McNutt. "The science is changing. Biogeoscience is an example."

Not everyone was in favour. Committee member Dork Sahagian, an Earth scientist at the University of New Hampshire, says that initially he was "dead set" against a new section, fearing it would "sequester" biological research. But he was soon persuaded it would integrate the disciplines. He then helped form, and presided over, a biogeoscience session on wetlands at last week's meeting.

Knauss says AGU officials have decided to "go back to the drawing boards" on a broader plan for reorganizing the existing ten sections. Robert Detrick, chairman of geology and geophysics at the Woods Hole Oceanographic Institution in Massachusetts, said the earlier plan was a complex formula to alter the scientific makeup of sections. He and others felt it was not sensitive enough to the needs of specific scientific disciplines.

"We need a mechanism to evolve over time," says Detrick, adding that more study is needed. A committee is being formed to work out an alternative plan. Rex Dalton 
- and evolution from the state curriculum, which local school districts look to for guidance (see Nature 400, 701; 1999). In New Mexico, however, scientists and their supporters managed to get Earth science and evolution reinstated after creationists had had them removed from the curriculum.

Speakers at last week's meeting cited Kansas as an example of the scientific community failing to be sufficiently involved in fighting creationists, while the New Mexico campaign was held up as a demonstration of the role scientists can play in the political process.

By talking to voters, researchers from institutions such as the University of New Mexico convinced them to elect nuclear physicist Marshall Berman of Sandia National Laboratories to the state school board. This voted in October to reinstate Earth science and evolution. "We can work in the trenches to improve the quality of science education," said John Geissman, a geologist from the University of New Mexico. "It is being done; it can be done."

Meanwhile, an organization called Kansas Citizens for Science is campaigning to replace three procreationism school board members who are coming up for re-election.

One teaching assistant at last week's meeting said she was trying to find out how to deal with students who had been imbued with creationist philosophy.

Also at the meeting were two procreationist AGU members. One of them, John Baumgardner, a geophysicist at Los Alamos National Laboratory in New Mexico, asked for a dialogue within the union on creationism. He said that creationists had been represented as "simplistic characters". But he offered no rebuttal when geophysicist Wilfred Elders, of the University of California at Riverside, criticized a creationist college text on the purported rapid formation of the Grand Canyon that cited some of Baumgardner's work. Rex Dalton

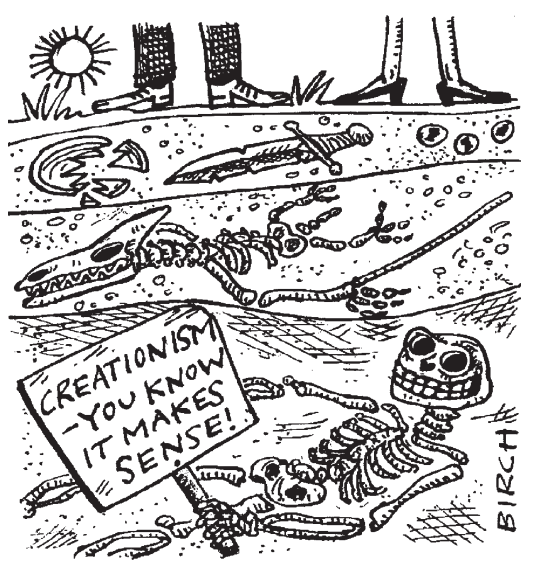

\section{Spain's universities split over merits of performance table}

\section{Barcelona}

A government-funded study that has ranked Spain's 44 public universities by giving each of them a score between one and ten has stirred up a fierce controversy.

Older universities came out best - seven of the top ten are at least 100 years old — and many of their officials say that the study confirms the quality of their work and shows the usefulness of comparative evaluation.

But younger universities, particularly those that are more technologically orientated, complain that the survey is based on outdated criteria. Young institutions accounted for eight of the ten universities that scored less than five.

The study was funded by the Ministry of Education and Culture. Scores were based on a survey by the National Statistics Institute. Six criteria were measured: educational development, organizational structure, teaching resources, participation of women, $\mathrm{PhD}$ activity and student success rate.

Carles Solà, rector of the top-scoring institution, the Autonomous University of Barcelona, says that his university's score of 8.5 "is congruent with previous data from diverse Spanish and European sources". He says that the evaluation process "may well open the doors to external observers in order to start objectives-based programmes".

Rafael Puyol, rector of the Complutense University of Madrid, which came second in the ranking, says that the result shows that, despite an "explosion" in student demand in recent years, "we have never overlooked quality". His university has more academic centres and students than any other in Spain.

The report has also been welcomed by Darío Villanueva, rector of the University of Santiago de Compostela, who says its results, "including our seventh position", are similar to those of an annual quality evaluation produced by a private company. The implication, he says, is that an evaluation culture already familiar in other countries "is beginning to emerge in Spain".

But Jaume Pagès, rector of the Polytechnic University of Catalonia in Barcelona, says the report is "inaccurate, unfinished, rash and lacking methodological rigour". According to Pagès, the quality of a university should be measured by whether "a series of internal and external objectives previously defined by the institution" have been reached - not through the use of indicators "arbitrarily established regardless of the university's goals".

He is particularly critical of the lack of external factors, such as those related to the social and economic environment or the

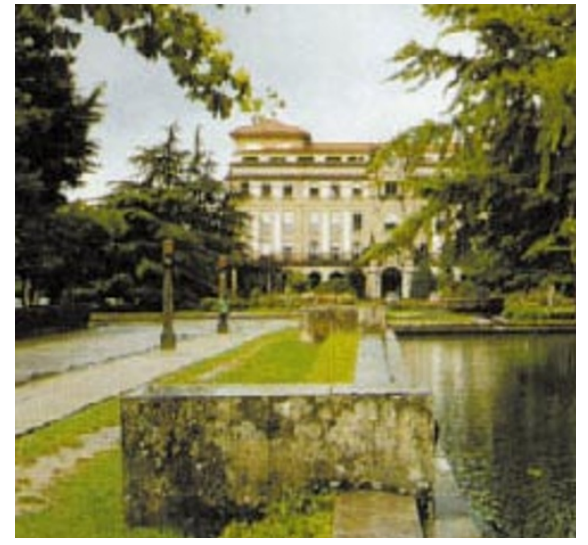

Seventh heaven? The University of Santiago de Compostela's rector is satisfied with its ranking.

number of students enrolled in international exchange programmes. Pagès says the report merely measures how close universities come to a "predefined, conventional, generalized and obsolete pattern", and adds that he is determined "to officially clarify problems involving quality in our universities".

Low-scoring universities have been especially critical of the failure to take account of links with private companies, for example through research contracts or patents.

Although the authors of the study say they were unable to include such a measure because of a lack of data, it appears to be a major reason why the 'technological' universities scored worse than expected.

The senior author of the study, Jesús de Miguel, director of the department of sociology and analysis of organizations at the University of Barcelona, and a social sciences consultant to the European Commission, has come under pressure from academics and the media. The rector of his university, Antoni Caparrós, has described the report as "not the work of my university".

Gemma Rauret, director of the public agency responsible for universities in Catalonia, points out that the report has not been submitted to experts' criticisms "but has sought media publicity”. However, says Rauret, "its most positive aspect is that it has triggered a rethinking of the need to supply more and better information".

In response to the study, Saturnino de la Plaza, president of the Council of Rectors of the Spanish Universities and rector of the Polytechnic University of Madrid, says that the council is to set up a commission that will evaluate the quality of universities but will not provide any type of ranking.

De la Plaza says the study was biased against technological institutions. "You can only compare like with like.” ～Xavier Bosch 